\title{
Preoperative Functional Status Is Associated With Unplanned Intubations Following Thyroidectomies
}

\author{
Rodney A Gabriel MD, Brittany N Burton MHS, Albert P Nguyen MD, and \\ Ulrich H Schmidt MD PhD MBA
}

\begin{abstract}
BACKGROUND: Unplanned postoperative intubation is an important event that may influence the outcome of thyroid- and parathyroidectomies. We performed a focused study on the association of preoperative functional status with unplanned intubation outcomes in these relatively common surgeries. METHODS: Utilizing data from the National Surgical Quality Improvement Program database from 2007 to 2013 , a propensity score-matched retrospective cohort study was performed assessing this outcome in the functionally independent versus dependent groups. Kaplan-Meier survival analysis and a Cox proportional hazards model were performed to assess the difference. RESULTS: There were a total of 98,035 thyroid- and parathyroidectomies identified from the National Surgical Quality Improvement Program from 2007 to 2013. After propensity score matching, there were 1,862 and 931 cases in the independent and dependent group, respectively. There were 11 versus 33 per 1,000 persons in the independent and dependent group, respectively, who experienced an unplanned intubation within $30 \mathrm{~d}$ following surgery $(P<.001)$. The dependent group showed worse intubation-free survival over $30 \mathrm{~d}(P<.001)$. There were no differences in this outcome during postoperative days $0-1(P=.17)$. Dependent functional status was statistically significantly associated with unplanned intubations up to $30 \mathrm{~d}$ postoperatively (hazard ratio 2.4 , 95\% CI 1.4-4.18, $P=.002)$. CONCLUSIONS: Preoperative functional status is a good marker for identifying patients at risk for re-intubation after thyroid- and parathyroidectomy. Key words: intubation; thyroidectomy; parathyroidectomy; respiratory insufficiency; frail elderly; perioperative care. [Respir Care 2017;62(10):1277-1283. (C) 2017 Daedalus Enterprises]
\end{abstract}

\section{Introduction}

It is well-established that unplanned postoperative reintubation is associated with increased morbidity and mortality and is an important quality and improvement measure. ${ }^{1,2}$ Accordingly, unplanned postoperative re-intubation is an important event that may influence the outcome of

\footnotetext{
The authors are affiliated with the Department of Anesthesiology, and Dr Gabriel is also affiliated with the Department of Biomedical Informatics, University of California San Diego, La Jolla, California.

This work was supported by National Library of Medicine Training Grant T15LM011271. The authors have disclosed no conflicts of interest.

Correspondence: Rodney A Gabriel MD, Department of Anesthesiology, University of California San Diego, 9500 Gilman Drive, MC 0881, La Jolla, CA 92093-0881. E-mail: ragabriel@ucsd.edu.
}

DOI: $10.4187 /$ respcare. 05575 thyroid- and parathyroidectomies. In this patient population, traditionally anesthesiologists focused on the emergent reintubation in the first $48 \mathrm{~h}$ post-surgery; however, respiratory failure later in the postoperative period has also been described.

In the United States, annually more than half of all surgical procedures are performed on those $65 \mathrm{y}$ and older. ${ }^{3}$ As such, there will be an increased demand for safe surgical procedures and unique challenges in caring for the elderly population. Therefore, it is essential that at-risk cohorts be identified. Current preoperative assessment tools often consider existing comorbidities; however, few of them consider the patient's physiologic reserve. ${ }^{4}$ Specifically, the term frailty is a concept that identifies patients (regardless of age) at increased risk of dying due to decline of physiologic reserve. ${ }^{5,6}$ The definition and quantification of a frailty index varies across studies; therefore, there is a lack of uniformity in its measurement. $4,5,7,8$ However, functional status, defined as the ability to independently 
perform activities of daily living, is an important component of its definition. Previous studies have shown that functional status is a measurable and effective variable in establishing preoperative risk. ${ }^{3}$ In contrast to frailty, functional disability is a relatively easier entity to define and measure.

The critical care literature has focused on the association between weakness and perioperative morbidity and mortality, including extubation failure. ${ }^{9-11}$ It has also been reported that surgical patients who are more frail have increased rates of perioperative mortality and complications. ${ }^{12}$ This includes patients undergoing thyroid and parathyroid surgery. ${ }^{13}$ Therefore, we performed a focused study on the association of low functional status with that of unplanned re-intubation outcomes in patients undergoing thyroid- and/or parathyroidectomies. We hypothesized that poor functional status at baseline would be associated with an increased unplanned intubation after this elective procedure.

\section{Methods}

\section{Data Collection}

Data were obtained from the publicly available data set of the American College of Surgeons National Surgical Quality Improvement Program (NSQIP) for the years 20072013. This is a multi-center, prospective, outcome-oriented database. Because the database is de-identified, it meets the criteria of the Health Insurance Portability and Accountability Act to protect personal information and was exempt from the consent requirement by our institutional review board. This database contains data from $>200$ participating hospitals for patients who underwent major surgical procedures. NSQIP undergoes a systemic sampling process called the 8-day cycle, which was developed to prevent bias in choosing cases for assessment. Data are collected via medical chart abstraction via a robust methodology. Every hospital facility participating in NSQIP has a trained and certified surgical clinical reviewer who captures data using a variety of methods, including medical chart abstraction. To ensure that the data collected are of the highest quality, NSQIP has provided various training mechanisms for the surgical clinical reviewers and conducts what is called an inter-rater reliability audit. The training mechanisms include a complete series of Webbased training modules followed by a certification exam (which must be retaken every year). The inter-rater reliability audit is a fundamental tool of NSQIP to assess validity of data among participating NSQIP sites. This involves an additional review of multiple charts, some of which are selected randomly and others selected based on various criteria of the chart to identify potential errors in the chart. An example of a selected chart based on criteria would include cases with 5 or more preoperative risk factors but no reported mortality or morbidity. The results of

\section{QUICK LOOK}

\section{Current knowledge}

Surgical patients undergoing thyroidectomies who are functionally dependent have increased rates of perioperative mortality and complications. Previous studies have demonstrated that functional dependence is associated with increased rates of unplanned intubations in this surgical population, who are at risk for unique airway complications - including recurrent laryngeal nerve injury, airway hematoma, and laryngospasm.

\section{What this paper contributes to our knowledge}

This study focused on the relationship between preoperative functional dependence and unplanned intubations in subjects who underwent thyroidectomy or parathyroidectomy. Whereas it demonstrated that there was an association with 30-d re-intubation rates, this was not the case during the first 2 hospital days, a time period when recurrent laryngeal nerve injury, airway hematoma, and laryngospasm are more likely to happen in post-thyroidectomy patients.

the inter-rater reliability audit reveal an overall disagreement between surgical clinical reviewer data at $<2 \%$, which is an acceptable rate per NSQIP standards. All thyroidectomies and parathyroidectomies were extracted from the database by identification via the surgical Common Procedural Terminology codes: 60210, 60220, 60225, 60240, 60252, 60254, 60260, 60270, 60271, 60500, and 60502. ${ }^{13}$

In this retrospective cohort study, the primary outcome of interest was postoperative unplanned intubation. In NSQIP, this is defined as the patient requiring placement of an endotracheal tube or other similar breathing tube (ie, supraglottic airway) and ventilator support within $30 \mathrm{~d}$ following surgery and was not intended or planned. This includes, but is not limited to, unplanned intubations for refractory hypotension, cardiac arrest, or inability to protect the airway; emergency tracheostomy; and accidental self-extubation requiring re-intubation. The postoperative day (from day 0 to 30) at which the initial unplanned intubation occurred is recorded. For patients who did not experience an unplanned intubation, data were censored after the number of days until discharge from hospital (up to day 30).

The primary exposure variable of interest was functional status. As described above, data were ascertained via medical chart abstraction from a trained surgical clinical reviewer and validated with additional reviews if necessary. This variable focuses on the patient's abilities to perform activities of daily living (ADLs) in the $30 \mathrm{~d}$ before surgery. ADLs were defined as activities usually performed in the course of a normal day, including feeding, bathing, 


\section{Preoperative Functional Status and Unplanned Intubations}

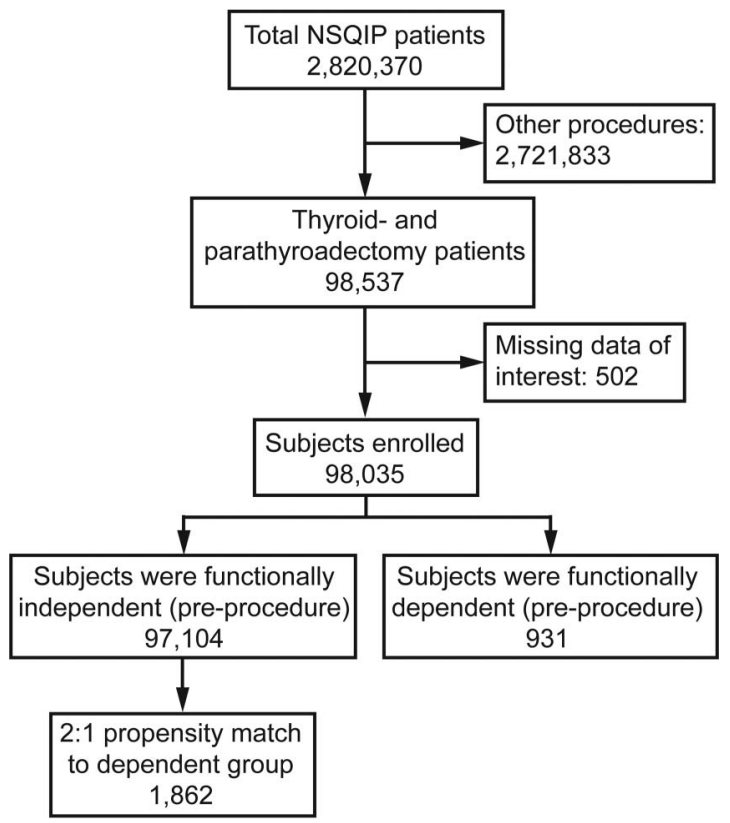

Fig. 1. Flow chart. NSQIP = National Surgical Quality Improvement Program.

toileting, dressing, and mobility. NSQIP categorizes this variable into independent (patient does not require any assistance from another person for any of the ADLs), partially dependent (patient requires some assistance from another person for ADLs), and totally dependent (patient requires total assistance for all ADLs). The best functional status demonstrated by the patient within the $30 \mathrm{~d}$ before surgery was reported. For the primary analysis of this study, this variable was treated as binary: either the patient was completely independent (ie, did not require assistance from another person for any activities of daily living) or dependent (ie, required some or total assistance from another person for activities of daily living). Confounding variables included in the analysis were age, body mass index (categorized as either $>40 \mathrm{~kg} / \mathrm{m}^{2}$ or not [to distinguish between those who are morbidly obese or not]), sex, smoking status (ie, whether the patient smoked within $1 \mathrm{y}$ of surgical admission), diabetes mellitus, COPD, congestive heart failure, hypertension, utilization of renal dialysis, chronic steroid use within $30 \mathrm{~d}$ of surgery, history of a bleeding disorder, and American Society of Anesthesiologists physical status classification score. These variables were chosen based on their potential association with postoperative reintubation. All cases with missing data for any of the above covariates were excluded from the analysis (Fig. 1).

\section{Statistical Analysis}

$\mathrm{R}$, a software environment for statistical computing ( $\mathrm{R}$ version 3.3.0), was used to perform all statistical analyses.
Pearson chi-square and Student $t$ tests were utilized to measure differences in demographic data between both cohorts (ie, independent vs dependent group). $P<.05$ was considered statistically significant. The MatchIt package ${ }^{14}$ for R statistical software was used for propensity score matching. MatchIt improves statistical models by preprocessing data with nonparametric matching methods. ${ }^{15} \mathrm{~A}$ 2:1 (independent/dependent) propensity score-matching method (using nearest-neighbor matching without replacement) was utilized to create matched cohorts. Because all covariates were statistically significantly different in both cohorts, the propensity score for being functionally dependent was determined using logistic regression based on body mass index, age, sex, smoking status, American Society of Anesthesiologists physical status class, and the presence of the following comorbidities: diabetes mellitus, COPD, congestive heart failure, hypertension, dialysis utilization, chronic steroid use, and bleeding disorder. To determine balance between matched groups, an absolute standardized difference $<0.1$ for each variable was considered adequate. For both the unmatched (including all subjects from the sample data, $n=98,035)$ and matched cohorts (only including matched subjects, $n=2,793$ ), a Kaplan-Meier survival analysis plot (survival $=$ free of unplanned intubation) was generated using the "survival" package from $\mathrm{R}$. The postoperative day (from days 0 to 30 ) in which the initial unplanned intubation occurred was calculated as the time-to-event. For subjects who did not experience an unplanned intubation, data were censored after the number of days until discharge from hospital (up to day 30). Differences in the survival curves for these 2 groups were compared using the log-rank test, in which $P<.05$ was considered statistically significant.

Using the matched data, a Cox proportional hazards model was used to estimate the association of functional status with unplanned intubation. Initially, this was done univariably for each covariate. Then all covariates that had a $P<.05$ were included in the multivariable model, which included the primary exposure variable and the propensity score for each case generated by the matching process described above. Next, another Cox proportional hazards model was developed on the total data set. For this model, the functional dependence variable was not dichotomized and, instead, was categorized into the 3 groups: functionally independent, partially dependent, and totally dependent (as described above). Hazard ratios (HRs) are reported with their associated 95\% CI. All HRs that do not include 1.00 in their 95\% CI were considered statistically significant.

\section{Results}

There were a total of 98,537 thyroid- and parathyroidectomies identified from NSQIP from 2007 to 2013. After exclusion, the sample size decreased to 98,035 , in which 
Table 1. Demographic Data of All Subjects From the National Surgical Quality Improvement Program Undergoing Thyroidectomy and Parathyroidectomy

\begin{tabular}{lccc}
\hline \hline \multicolumn{1}{c}{ Characteristics } & Independent & Dependent & $P$ \\
\hline Total, $n$ & 97,104 & 931 & \\
Unplanned intubations, $n(\%)$ & $314(0.3)$ & $31(3.3)$ & $<.001$ \\
Age, mean \pm SD y & $53.8 \pm 1.5$ & $64.9 \pm 1.6$ & $<.001$ \\
BMI $>40 \mathrm{~kg} / \mathrm{m}^{2}, n(\%)$ & $10,255(10.6)$ & $126(13.5)$ & .004 \\
Male sex, $n(\%)$ & $20,510(21.1)$ & $246(26.4)$ & $<.001$ \\
Smoker, $n(\%)$ & $13,735(14.1)$ & $107(11.5)$ & .02 \\
Diabetes mellitus, $n(\%)$ & $12,086(12.4)$ & $249(26.7)$ & $<.001$ \\
COPD, $n(\%)$ & $1,957(2.0)$ & $93(10.0)$ & $<.001$ \\
CHF, $n(\%)$ & $212(0.2)$ & $36(3.9)$ & $<.001$ \\
Hypertension, $n(\%)$ & $42,174(43.4)$ & $636(68.3)$ & $<.001$ \\
Dialysis, $n(\%)$ & $1,888(1.9)$ & $127(13.6)$ & $<.001$ \\
Steroid use, $n(\%)$ & $2,152(2.2)$ & $63(6.8)$ & $<.001$ \\
ASA PS III-V, $n$ (\%) & $29,298(30.2)$ & $768(82.5)$ & $<.001$ \\
Bleeding disorder, $n(\%)$ & $1,432(1.5)$ & $80(8.6)$ & $<.001$ \\
& & & \\
\hline The 2 cohorts are the functionally independent and dependent groups. & \\
BMI = body mass index & & & \\
CHF $=$ congestive heart failure & & \\
ASA PS = American Society of Anesthesiologists physical status & & \\
\hline
\end{tabular}

931 were in the dependent cohort. Within the independent and dependent cohorts, the crude rate for unplanned postoperative 30-d intubation was 3 versus 33 per 1,000 persons, respectively (difference in rates, $P<.001$ ). Among all covariates, there were statistically significant differences between both cohorts (Table 1). During postoperative days $0-1$, the independent and dependent cohorts had crude unplanned intubation rates of 2 versus 11 per 1,000 persons, respectively $(P<.001)$. In the unadjusted samples, the independent cohort had improved 30-d unplanned intubation-free survival compared with the dependent cohort (Fig. 2, $P<.001$ ).

Subsequently, 2:1 propensity score matching was employed. There were no differences in the covariates between the matched cohorts based on standardized mean differences across all covariates $<0.1$ (Table 2). Within the independent and dependent cohorts, 11 versus 33 per 1,000 persons, respectively, had an unplanned intubation postoperatively within $30 \mathrm{~d}$ (difference in rates, $P<.001$ ). During postoperative days $0-1$, there was no statistically significant difference in unplanned intubations between the cohorts. The independent and dependent cohorts had similar unplanned intubations, with rates of 5 versus 11 per 1,000 persons during this time period, respectively $(P=.17)$. However, in the 30 -d period, the independent cohort had improved unplanned intubation-free survival compared with the dependent cohort (Fig. 3, $P<.001$ ).

In a Cox proportional hazards model in which the calculated propensity score from the matching process and functional status was used to predict unplanned intubations, functional dependence was associated with this out-

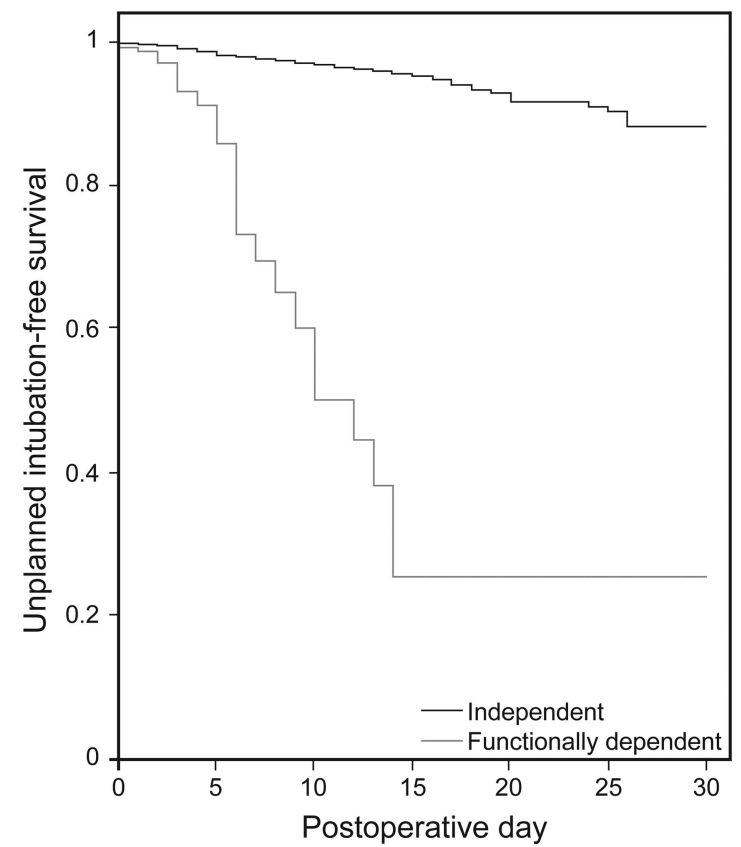

Fig. 2. Kaplan-Meier survival analysis for unplanned intubations following thyroidectomy and parathyroidectomy using all data (unadjusted).

come (HR 2.39, 95\% CI 1.36-4.18, $P=.002$ ). A separate Cox proportional hazards model was performed in which all study data were used and functional status was categorized into functionally independent, partially dependent, and totally dependent. All of the covariates, including the primary exposure of interest, afforded statistically significant HRs from the univariable analysis (Table 3). All covariates were then included in the final multivariable model building. When compared with functionally independent subjects, both the partially dependent (HR 3.06, 95\% CI 1.94-4.83, $P<.001)$ and totally dependent groups (HR 4.58, 95\% CI 2.20-9.55, $P<.001$ ) were associated with unplanned intubations.

\section{Discussion}

In this retrospective cohort study analyzing longitudinal NSQIP data from patients undergoing thyroid- and/or parathyroidectomies, we demonstrated that preoperative functional disability was associated with 30-d unplanned intubations after surgery. This was true after controlling for several comorbidities (eg, diabetes, COPD, etc) and demographic data (age, sex, body mass index) via propensity score-matched cohorts. Unplanned re-intubation following surgery, although relatively uncommon, is associated with increased mortality and morbidity; therefore, identifying patients at higher risk for even low-to-moderate-risk surgeries, such as thyroidectomies, is prudent. 
Table 2. Demographic Data of Propensity-Matched Cohorts Undergoing Thyroidectomy and Parathyroidectomy

\begin{tabular}{|c|c|c|c|c|}
\hline Characteristics & Independent & Dependent & $P$ & SMD \\
\hline Total, $n$ & 1,862 & 931 & & \\
\hline Unplanned intubations, $n(\%)$ & $21(1.1)$ & $31(3.3)$ & $<.001$ & \\
\hline Age, mean $\pm \mathrm{SD}$ y & $65.6 \pm 1.5$ & $64.9 \pm 1.6$ & .26 & 0.044 \\
\hline $\mathrm{BMI}>40 \mathrm{~kg} / \mathrm{m}^{2}, n(\%)$ & $243(13.1)$ & $126(13.5)$ & .77 & 0.014 \\
\hline Male sex, $n(\%)$ & $506(27.2)$ & $246(26.4)$ & .71 & 0.008 \\
\hline Smoker, $n(\%)$ & $179(9.6)$ & $107(11.5)$ & .14 & 0.019 \\
\hline Diabetes mellitus, $n(\%)$ & $506(27.2)$ & $249(26.7)$ & .84 & 0.004 \\
\hline COPD, $n(\%)$ & $185(9.9)$ & $93(10.0)$ & .99 & 0.001 \\
\hline $\mathrm{CHF}, n(\%)$ & $60(3.2)$ & $36(3.9)$ & .44 & 0.006 \\
\hline Hypertension, $n(\%)$ & $1,287(69.1)$ & $636(68.3)$ & .69 & 0.008 \\
\hline Dialysis, $n(\%)$ & $256(13.7)$ & $127(13.6)$ & .98 & 0.001 \\
\hline Steroid use, $n(\%)$ & $100(5.4)$ & $63(6.8)$ & .16 & 0.014 \\
\hline ASA PS III-V, $n(\%)$ & $1,528(82.1)$ & $768(82.5)$ & .82 & 0.004 \\
\hline Bleeding disorder, $n(\%)$ & $136(7.3)$ & $80(8.6)$ & .26 & 0.014 \\
\hline $\begin{array}{l}\text { The } 2 \text { cohorts are the functionally indepe } \\
\text { SMD = standardized mean difference } \\
\text { BMI }=\text { body mass index } \\
\text { CHF }=\text { congestive heart failure } \\
\text { ASA PS = American Society of Anesthe }\end{array}$ & & & & \\
\hline
\end{tabular}

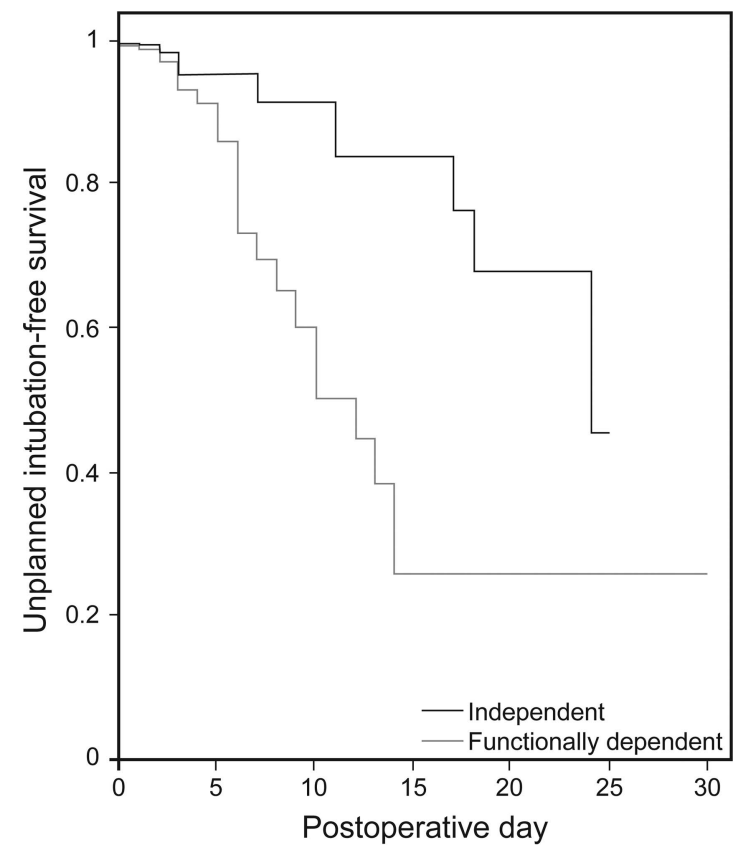

Fig. 3. Adjusted Kaplan-Meier survival analysis for unplanned intubations following thyroidectomy and parathyroidectomy using propensity-matched cohorts.

To our knowledge, this is the first study to assess the impact of functional disability on postoperative re-intubation rates in this surgical group using matched cohorts. In contrast to a previous study using NSQIP that identified multiple predictors associated with unplanned intubations after thyroid/parathyroidectomies, ${ }^{13}$ the current study focused on a specific preoperative characteristic, functional status. This is defined as the dependence of the patient on others to help with activities of daily living. This is an important marker for increased risk of surgical mortality and morbidity, as has been demonstrated in many studies assessing frailty indices and surgical outcome., , $, 5,7,16$ Functional dependence is a preoperative marker related to 6-month mortality in geriatric surgery that requires postoperative ICU admission. ${ }^{17}$ Furthermore, functional status has been demonstrated to be associated with unplanned postoperative intubations and respiratory failure in various NSQIP studies looking at all major noncardiac surgeries, neurosurgery, and complex general and vascular surgery. ${ }^{13,18-20}$

Thyroid- and parathyroidectomies are not high-risk surgical procedures $;{ }^{21}$ however, there are complications unique to this patient population, since airway compromise due to respiratory failure related to surgical hematoma, ${ }^{22,23}$ recurrent laryngeal nerve injury, and hypocalcemia-induced laryngospasm are possible. ${ }^{24}$ Usually, these specific surgical complications occur within the first $48 \mathrm{~h}$. Our data show that there was no difference in unplanned intubation rates between matched cohorts during the first 2 hospital days. This supports the point that early re-intubation in this patient population is probably more due to surgical complications. In contrast, overall $30-\mathrm{d}$ intubation-free survival was worse in the dependent group. This suggests that it is not necessarily just the unique airway complications associated with thyroidectomies that functionally dependent individuals are at increased risk for, but rather overall complications associated with respiratory failure. 
Table 3. Results of the Cox Proportional Hazards Model

\begin{tabular}{|c|c|c|c|c|}
\hline \multirow{2}{*}{ Characteristics } & \multicolumn{2}{|c|}{ Unadjusted } & \multicolumn{2}{|c|}{ Adjusted } \\
\hline & HR $(95 \% \mathrm{CI})$ & $P$ & HR $(95 \% \mathrm{CI})$ & $P$ \\
\hline \multicolumn{5}{|c|}{ Dependent functional status } \\
\hline Independent & Reference & & Reference & \\
\hline Partially dependent & $7.63(4.95-11.76)$ & $<.001$ & $3.06(1.94-4.83)$ & $<.001$ \\
\hline Totally dependent & $14.59(7.50-28.39)$ & $<.001$ & $4.58(2.20-9.55)$ & $<.001$ \\
\hline Age (per decade) & $1.35(1.25-1.45)$ & $<.001$ & $1.17(1.08-1.28)$ & $<.001$ \\
\hline Morbid obesity & $1.38(1.01-1.87)$ & .042 & $0.99(0.72-1.37)$ & .96 \\
\hline Male sex & $1.31(1.03-1.67)$ & .03 & $0.99(0.78-1.27)$ & .98 \\
\hline Smoker & $1.49(1.14-1.94)$ & .003 & $1.53(1.16-2.03)$ & .003 \\
\hline Diabetes mellitus II & $2.19(1.71-2.81)$ & $<.001$ & $1.21(0.93-1.58)$ & .15 \\
\hline COPD & $4.54(3.14-6.56)$ & $<.001$ & $1.92(1.30-2.83)$ & .001 \\
\hline $\mathrm{CHF}$ & $9.23(4.92-17.34)$ & $<.001$ & $1.81(0.89-3.65)$ & .10 \\
\hline Hypertension & $2.53(2.03-3.12)$ & $<.001$ & $1.26(0.98-1.64)$ & .08 \\
\hline Dialysis & $4.46(3.10-6.41)$ & $<.001$ & $2.09(1.38-3.14)$ & $<.001$ \\
\hline Steroid use & $2.23(1.39-3.59)$ & $<.001$ & $1.23(0.75-2.00)$ & .41 \\
\hline ASA PS III-V & $4.30(3.44-5.37)$ & $<.001$ & $2.51(1.93-3.27)$ & $<.001$ \\
\hline Bleeding disorder & $4.84(3.24-7.23)$ & $<.001$ & $2.12(1.39-3.23)$ & $<.001$ \\
\hline \multicolumn{5}{|l|}{$\begin{array}{l}\mathrm{HR}=\text { hazard ratio } \\
\mathrm{BMI}=\text { body mass index } \\
\mathrm{CHF}=\text { congestive heart failure } \\
\text { ASA PS = American Society of }\end{array}$} \\
\hline
\end{tabular}

The specific pathophysiological mechanism at which functional disability may cause increased surgical/anesthesia complications is unclear; however, it is logical to assume that functional ability provides information about a patient's comorbidity burden and physiological reserve. ${ }^{25}$ Physical capacity can decline by as much as $40 \%$ in an uncomplicated operation. ${ }^{26}$ It is possible that the toll from the surgical insult coupled with a poor functional status limits the patient's ability to tolerate further stressors. Overall muscle weakness has been reported to be correlated with pharyngeal dysfunction, increasing the rate of aspiration and respiratory failure. ${ }^{9,11}$ Muscle weakness has also been associated with re-intubation in a general surgical ICU population. Therefore, it is essential that a comprehensive preoperative evaluation by the surgeon and anesthesiologist include not only the presence of various comorbidities but also the presence of functional disability, because this one variable may paint a larger picture of how much their comorbidities are affecting their daily living. In the presence of functional disability in a patient who will undergo thyroidectomy, preoperative rehabilitation may be beneficial.

Preoperative rehabilitation, or prehabilitation, is the concept of enhancing physical function before surgery and has been specifically aimed at the elderly. ${ }^{26}$ The centerpiece of prehabilitation is exercise training starting $6-8$ weeks before surgery with the intention of improving cardiopulmonary function. ${ }^{27,28}$ This concept has been applied to those undergoing elective colorectal, cardiac, and orthopedic surgeries. ${ }^{26-29}$ A meta-analysis on prehabilitation demonstrated shortened hospital stays and modest improvement on postsurgical physical status. ${ }^{30}$ In the presence of malignancies of the thyroid and parathyroid, the risk of delaying the case versus the benefits of rehabilitation requires careful consideration, because the latter may not allow enough time to prevent progression of disease. However there is evidence that direct postoperative mobilization even in ICU patients improves functional outcome and decreases stay. ${ }^{10}$ Identifying patients at risk for postoperative respiratory failure and re-intubation might allow the clinician to develop a patient-centered strategy to minimize these complications.

There are some major limitations to the study. First, we are investigating unplanned postoperative intubations in a surgical population that is at risk for unique airway complications, including surgical hematomas, nerve injury, and laryngospasm. NSQIP does not provide data as to the event that triggered the intubation; therefore, we are unable to differentiate between surgery-specific causes or more general medical causes of respiratory failure leading to intubation. Because these unique airway complications generally occur earlier in the postoperative period, re-intubation rates during the first $2 \mathrm{~d}$ and overall $30-\mathrm{d}$ mortality were separately analyzed. In addition, NSQIP does not include other comorbidities that may influence re-intubation rates, including obstructive sleep apnea. The inherent nature of this large database research introduces limitations related to retrospective study designs as well. Furthermore, one of the problems with propensity matching is the problem of missing data, in which consequently you can only include cases with 


\section{Preoperative Functional Status and Unplanned Intubations}

complete information. However, after removing all cases with missing data, the total sample size only decreased from 98,537 to 98,035 , which is about $0.5 \%$ of the data.

\section{Conclusions}

In conclusion, based on the presented results, preoperative functional status is a good marker for identifying those at risk for unplanned re-intubations following thyroid- and/or parathyroidectomies. It is important to evaluate the independence of functional status in patients undergoing thyroid and parathyroid surgeries. Knowledge of the increased risk of unplanned intubations in this cohort should be anticipated and included during the informed consent process.

\section{REFERENCES}

1. Ramachandran SK, Nafiu OO, Ghaferi A, Tremper KK, Shanks A, Kheterpal S. Independent predictors and outcomes of unanticipated early postoperative tracheal intubation after nonemergent, noncardiac surgery. Anesthesiology 2011;115(1):44-53.

2. Tillquist MN, Gabriel RA, Dutton RP, Urman RD. Incidence and risk factors for early postoperative reintubations. J Clin Anesth 2016; 31:80-89.

3. Oresanya LB, Lyons WL, Finlayson E. Preoperative assessment of the older patient: a narrative review. JAMA 2014;311(20):21102120 .

4. Revenig LM, Canter DJ, Taylor MD, Tai C, Sweeney JF, Sarmiento $\mathrm{JM}$, et al. Too frail for surgery? Initial results of a large multidisciplinary prospective study examining preoperative variables predictive of poor surgical outcomes. J Am Coll Surg 2013;217(4):665670.e1.

5. Hall DE, Arya S, Schmid KK, Blaser C, Carlson MA, Bailey TL, et al. Development and initial validation of the risk analysis index for measuring frailty in surgical populations. JAMA Surg 2017;152(2): 175-182.

6. Heppenstall CP, Wilkinson TJ, Hanger HC, Keeling S. Frailty: dominos or deliberation? N Z Med J 2009;122(1299):42-53.

7. Theou O, Brothers TD, Mitnitski A, Rockwood K. Operationalization of frailty using eight commonly used scales and comparison of their ability to predict all-cause mortality. J Am Geriatr Soc 2013; 61(9):1537-1551.

8. Theou O, Brothers TD, Peña FG, Mitnitski A, Rockwood K. Identifying common characteristics of frailty across seven scales. J Am Geriatr Soc 2014;62(5):901-906.

9. Mirzakhani H, Williams JN, Mello J, Joseph S, Meyer MJ, Waak K, et al. Muscle weakness predicts pharyngeal dysfunction and symptomatic aspiration in long-term ventilated patients. Anesthesiology 2013;119(2):389-397.

10. Schaller SJ, Anstey M, Blobner M, Edrich T, Grabitz SD, GradwohlMatis I, et al. Early, goal-directed mobilisation in the surgical intensive care unit: a randomised controlled trial. Lancet 2016;388(10052): 1377-1388

11. Piriyapatsom A, Williams EC, Waak K, Ladha KS, Eikermann M, Schmidt UH. Prospective observational study of predictors of reintubation following extubation in the surgical ICU. Respir Care 2016;61(3):306-315.

12. Adams P, Ghanem T, Stachler R, Hall F, Velanovich V, Rubinfeld I. Frailty as a predictor of morbidity and mortality in inpatient head and neck surgery. JAMA Otolaryngol Head Neck Surg 2013;139(8): 783-789.
13. Gupta PK, Gupta H, Natarajan B, Shetty S, Smith RB, Lindau R, 3rd, et al. Postoperative respiratory failure after thyroid and parathyroid surgery: analysis of national surgical quality improvement program. Head Neck 2012;34(3):321-327.

14. Ho D, Imai K, Imai MK. Package "MatchIt". Version 2.4. https:// cran.r-project.org/web/packages/MatchIt/MatchIt.pdf. Accessed June 2013.

15. Ho DE, Imai K, King G, Stuart EA. Matching as nonparametric preprocessing for reducing model dependence in parametric causal inference. Political Anal 2007;15(3):199-236.

16. Makary MA, Segev DL, Pronovost PJ, Syin D, Bandeen-Roche K, Patel P, et al. Frailty as a predictor of surgical outcomes in older patients. J Am Coll Surg 2010;210(6):901-908.

17. Robinson TN, Eiseman B, Wallace JI, Church SD, McFann KK, Pfister SM, et al. Redefining geriatric preoperative assessment using frailty, disability and co-morbidity. Ann Surg 2009;250(3):449-455.

18. Arozullah AM, Daley J, Henderson WG, Khuri SF. Multifactorial risk index for predicting postoperative respiratory failure in men after major noncardiac surgery. The National Veterans Administration Surgical Quality Improvement Program. Ann Surg 2000;232(2):242-253.

19. Cote DJ, Karhade AV, Burke WT, Larsen AM, Smith TR. Risk factors for post-operative respiratory failure among 94,621 neurosurgical patients from 2006 to 2013: a NSQIP analysis. Acta Neurochir 2016;158(9):1639-1645.

20. Scarborough JE, Bennett KM, Englum BR, Pappas TN, LagooDeenadayalan SA. The impact of functional dependency on outcomes after complex general and vascular surgery. Ann Surg 2015; 261(3):432-437.

21. Meltzer C, Klau M, Gurushanthaiah D, Titan H, Meng D, Radler L, Sundang A. Risk of complications after thyroidectomy and parathyroidectomy: a case series with planned chart review. Otolaryngol Head Neck Surg 2016;155(3):391-401.

22. Suzuki S, Yasunaga H, Matsui H, Fushimi K, Saito Y, Yamasoba T. Factors associated with neck hematoma after thyroidectomy: a retrospective analysis using a Japanese inpatient database. Medicine 2016;95(7):e2812.

23. Dixon JL, Snyder SK, Lairmore TC, Jupiter D, Govednik C, Hendricks JC. A novel method for the management of post-thyroidectomy or parathyroidectomy hematoma: a single-institution experience after over 4,000 central neck operations. World J Surg 2014; 38(6): 1262-1267.

24. Bhattacharyya N, Fried MP. Assessment of the morbidity and complications of total thyroidectomy. Arch Otolaryngol Head Neck Surg 2002;128(4):389-392.

25. Ponzetto M, Maero B, Maina P, Rosato R, Ciccone G, Merletti F, et al. Risk factors for early and late mortality in hospitalized older patients: the continuing importance of functional status. J Gerontol A Biol Sci Med Sci 2003;58(11):1049-1054.

26. Mayo NE, Feldman L, Scott S, Zavorsky G, Kim DJ, Charlebois P, et al. Impact of preoperative change in physical function on postoperative recovery: argument supporting prehabilitation for colorectal surgery. Surgery 2011;150(3):505-514.

27. Nielsen PR, Jørgensen LD, Dahl B, Pedersen T, Tønnesen H. Prehabilitation and early rehabilitation after spinal surgery: randomized clinical trial. Clin Rehabil 2010;24(2):137-148.

28. Hoogeboom TJ, Dronkers JJ, Hulzebos EH, van Meeteren NL. Merits of exercise therapy before and after major surgery. Curr Opin Anaesthesiol 2014;27(2):161-166.

29. Furze G, Dumville JC, Miles JN, Irvine K, Thompson DR, Lewin RJ. "Prehabilitation" prior to CABG surgery improves physical functioning and depression. Int J Cardiol 2009;132(1):51-58.

30. Santa Mina D, Clarke H, Ritvo P, Leung YW, Matthew AG, Katz J, et al. Effect of total-body prehabilitation on postoperative outcomes: a systematic review and meta-analysis. Physiotherapy 2014;100(3):196-207. 